\title{
A general approach for investigating enzymatic pathways and substrates for ubiquitin-like modifiers
}

\author{
Tianwei Li ${ }^{\mathrm{a}, 1}$, Rasa Santockyte ${ }^{\mathrm{b}, 1}$, Rong-Fong Shen ${ }^{\mathrm{c}}$, Ephrem Tekle ${ }^{\mathrm{a}}$, Guanghui Wang ${ }^{\mathrm{c}}$, \\ David C.H. Yang ${ }^{b}$, P. Boon Chock ${ }^{a, *}$ \\ ${ }^{a}$ Laboratory of Biochemistry, National Heart, Lung, and Blood Institute, National Institutes of Health, Bethesda, MD 20892, USA \\ ${ }^{\mathrm{b}}$ Department of Chemistry, Georgetown University, Washington, DC 20057, USA \\ ${ }^{\mathrm{c}}$ Proteomics Core Facility, National Heart, Lung, and Blood Institute, National Institutes of Health, Bethesda, MD 20892, USA
}

Received 2 March 2006

Available online 20 March 2006

\begin{abstract}
Ubiquitin-like modifiers (UBLs) contain ubiquitin homology domains and can covalently modify target proteins in a manner similar to ubiquitylation. In this study, we revealed a general proteomic approach to elucidate the enzymatic pathways and identify target proteins for three UBLs: SUMO-2, SUMO-3, and NEDD8. Expression plasmids containing the cDNAs of Myc/6× His doubly-tagged processed or non-conjugatable forms of these UBLs were constructed. The constructed vectors were then used to transfect HEK 293 Tet-On cells, and stable cell lines expressing these UBLs and their mutants were established. The epitope-tagged proteins were purified by immunoprecipitation under native conditions or by affinity chromatography on nickel resin under denaturing conditions. Purified proteins were analyzed using liquid chromatography coupled with mass spectrometry (LC-MS/MS). Most of the E1-like activating enzymes, E2like conjugating enzymes and the majorities of the known target as well as some previously unreported proteins for SUMO-2, SUMO-3, and NEDD8 pathways were identified.
\end{abstract}

Published by Elsevier Inc.

Keywords: Ubiquitin-like modifiers; SUMO; NEDD8; Mass spec; Proteomics

Posttranslational modification of proteins plays important roles in regulating the functions of target proteins [1]. Ubiquitin is known to covalently modify its target proteins and alter their stabilities, activities, and localizations [2]. The conjugation of target proteins is carried out through an enzymatic cascade, consisting of ubiquitin-activating enzyme (E1), ubiquitin-conjugating enzyme (E2), and ubiquitin ligases (E3) [3,4], that covalently links the ubiquitin C-terminal glycine residue to one or more specific lysine residue(s) of the target proteins. This process can be reversed by the action of deubiquitinating enzymes, which remove ubiquitin moieties from the modified proteins [5]. Polyubiquitylated (at least four ubiquitin moieties) proteins

\footnotetext{
* Corresponding author. Fax: +1 3014960599.

E-mail address: BChock@nih.gov (P.B. Chock).

${ }^{1}$ These authors contributed equally to this work.
}

are targeted for selective degradation by the $26 \mathrm{~S}$ proteasome [6]. In addition, ubiquitylation also plays important roles in other cellular functions, such as DNA repair, and transcription regulation and endocytosis [7-9].

Recently, a growing number of proteins containing domains with significant homology to ubiquitin have been identified. These ubiquitin-like proteins can be classified into two groups, based on their abilities to covalently modify target proteins. The ubiquitin-domain proteins (UDPs) such as RAD23 or Gdx [10] contain embedded ubiquitinlike domains, but fail to form conjugated substrates while the ubiquitin-like modifiers (UBLs), ${ }^{2}$ which are small

\footnotetext{
${ }^{2}$ Abbreviations used: UBL, ubiquitin-like protein; SUMO, small ubiquitin-related modifier; pRB, retinoblastoma tumor suppressor protein; NEDD8, Neuronal-precursor-cell-expressed developmentally downregulated protein-8.
} 
polypeptides consisting of either one or two ubiquitin homologous domains, can covalently conjugate to their target proteins in a manner similar to ubiquitylation. These proteins include SUMO, NEDD8, ISG15, FAT10, URM1, etc. [11-13]. Similar to ubiquitin, these ubiquitin-like modifiers (UBLs) are small proteins capable of covalently modifying target proteins and altering their functions [14]. Functional characterization of protein modification by ubiquitin and UBLs greatly depends on identifications of both enzymatic pathways and the target proteins.

In the last decade, the enzymatic pathways and relatively abundant substrates were discovered for SUMO, NEDD8, and ISG15, thank to the efforts of several groups of investigators using biochemical and genetic approaches [15-19]. The discoveries of these enzymes and protein substrates significantly enhance our understanding of the functions of these UBLs. However, the enzymatic pathways and major substrates for FAT10 and URM1, etc. remain unclear $[20,21]$. Herein, we report a general proteomic approach for the identification of both the enzymatic cascades and major substrates using three UBLs (SUMO-2, SUMO-3, and NEDD8) as examples. This method is fast and efficient, and offers a promising alternative for the investigation of UBL functions. Mass spectrometry-based proteomic methods have proven useful for identifying the target proteins for ubiquitin, SUMO, and ISG15 [22-25].

\section{Materials and methods}

\section{Antibodies, plasmids, and mutagenesis}

Monoclonal anti-Myc (9E10), anti-CUL1, anti-c-Jun, and anti-p53 (DO-1) antibodies, agarose conjugated antiMyc antibody, TRITC conjugated anti-Myc and FITC conjugated anti-PML antibodies were purchased from Santa Cruz Biotechnology Inc. FITC conjugated goat antimouse IgG antibody was purchased from Sigma. The cDNAs encoding the processed forms of SUMO-2 (1-92), SUMO-3 (1-93), and NEDD8 (1-76) with GlyGly at their C-termini were amplified by PCR. $6 \times$ His-tag sequences immediately upstream of the start codon of the SUMO-2/3 and NEDD8 sequences were designed in the amplifying primers. The PCR amplified cDNAs were inserted into the pTRE2hyg2-Myc vector (Clontech) as NheI/ClaI fragments to generate pTRE2hyg2-Myc-His-SUMO-2/3GG and pTRE2hyg2-Myc-His-NEDD8GG plasmids. The pTRE2hyg2-Myc-His-SUMO-2/3 $\Delta$ GG and pTRE2hyg2Myc-His-NEDD8 $\Delta$ GG plasmids harboring non-conjugatable SUMO-2 (1-90), SUMO-3 (1-91), and NEDD8 (1-74) sequences, respectively, lacking the C-terminal GlyGly, were similarly constructed.

\section{Cell Culture, transfection, and immunofluorescence}

The stable HEK 293 Tet-On cell lines overexpessing Myc-His-SUMO-2/3GG, Myc-His-NEDD8GG, Myc-HisSUMO-2/3 $\Delta \mathrm{GG}$, and Myc-His-NEDD8 $\Delta \mathrm{GG}$ were established using the protocol previously described [22]. FuGENE6 (Roche) was used for transfection. For immunofluorescence, HEK 293 Tet-On cells harboring pTRE2hyg2-Myc-His-SUMO-2/3GG and pTRE2hyg2Myc-His-NEDD8GG were seeded onto 8-well Lab-Tek chamber slide (Nalge Nuc International) and induced with $2 \mu \mathrm{g} / \mathrm{ml}$ Dox for $48 \mathrm{~h}$. Cells were fixed in $3.7 \%$ formaldehyde and permeablized with $0.1 \%$ Triton X-100. For single-antibody staining, the fixed cells were incubated with anti-Myc primary antibody for $1 \mathrm{~h}$, and then the slide was rinsed with $1 \times$ PBS and incubated with FITC conjugated goat antimouse IgG secondary antibody for $1 \mathrm{~h}$. The cells were then incubated with propidium iodide (PI) for $5 \mathrm{~min}$ and washed 5 times with $1 \times$ PBS. For double-antibody staining, the fixed cells were first stained with TRITC conjugated antiMyc antibody and washed 5 times with $1 \times$ PBS, then stained with FITC conjugated anti-PML antibody and washed with $1 \times$ PBS as described above. Images were captured on a Zeiss LSM-5 confocal microscope.

\section{Purification of $6 \times$ His-tagged UBL substrates}

The $6 \times$ His-tagged proteins were purified under denaturing conditions using Ni-NTA agarose according to the manufacturer's instructions (Qiagen) with modifications. Briefly, cells stably expressing Myc-His-UBL-GGs and Myc-His-UBL $\Delta$ GGs were induced with $2 \mu \mathrm{g} / \mathrm{ml}$ doxycycline (Dox) for $48 \mathrm{~h}$. To enhance the level of NEDD8 conjugated proteins, $5 \mu \mathrm{M}$ MG132 was added to cells expressing Myc-His-NEDD8GG for $16 \mathrm{~h}$ before harvesting. One hundred million cells were lysed in lysis buffer A [6 $\mathrm{M}$ guanidinium- $\mathrm{HCl}\left(\mathrm{pH}\right.$ 8.0), $100 \mathrm{mM} \mathrm{Na} \mathrm{NaPO}_{4} /$ $\mathrm{NaH}_{2} \mathrm{PO}_{4}, 10 \mathrm{mM}$ Tris- $\mathrm{HCl}, 10 \mathrm{mM}$ imidazole, $10 \mathrm{mM}$ $\beta$-mercaptoethanol, $20 \mathrm{mM} \mathrm{N}$-ethylmaleimide, and $20 \mathrm{mM}$ iodoacetamide]. The clarified cell lysates were incubated with Ni-NTA agarose beads for $1 \mathrm{~h}$ at $4{ }^{\circ} \mathrm{C}$. The beads were washed twice, each time for $2 \mathrm{~min}$, with $0.5 \mathrm{ml}$ of each of the following buffers: lysis buffer $\mathrm{A}$, washing buffer $\mathrm{A}$ [8 $\mathrm{M}$ urea ( $\mathrm{pH} 8.0), 100 \mathrm{mM} \mathrm{Na} 2 \mathrm{HPO}_{4} / \mathrm{NaH}_{2} \mathrm{PO}_{4}, 10 \mathrm{mM}$ Tris- $\mathrm{HCl}, 10 \mathrm{mM}$ imidazole, and $20 \mathrm{mM} N$-ethylmaleimide and washing buffer $\mathrm{B}[8 \mathrm{M}$ urea $(\mathrm{pH} 6.3), 100 \mathrm{mM}$ $\mathrm{Na}_{2} \mathrm{HPO}_{4} / \mathrm{NaH}_{2} \mathrm{PO}_{4}, 10 \mathrm{mM}$ Tris- $\mathrm{HCl}, 10 \mathrm{mM}$ imidazole, $20 \mathrm{mM} N$-ethylmaleimide, and $0.2 \%$ Triton X]. For Western blotting, the $6 \times$ His-tagged proteins were eluted by boiling Ni-NTA agarose beads in $2 \mathrm{NuPAGE}$ (Invitrogen) sample buffer containing $250 \mathrm{mM}$ imidazole. Protein samples were resolved by $4-12 \%$ NuPAGE gels and probed with specific antibodies.

\section{Immunoprecipitation and immunoblotting}

For immunoprecipitation, cells were lysed in lysis buffer B [50 mM Tris- $\mathrm{HCl}$ (pH 7.5), $150 \mathrm{mM} \mathrm{NaCl}, 1 \%$ Nonidet P-40, $5 \mathrm{mM}$ EDTA, $20 \mathrm{mM} N$-ethylmaleimide, $1 \mathrm{mM}$ PMSF, $10 \mu \mathrm{g} / \mathrm{ml}$ pepstatin, $20 \mu \mathrm{g} / \mathrm{ml}$ leupeptin, and $10 \mu \mathrm{g} / \mathrm{ml}$ aprotinin] to obtain whole cell extracts. Immunoprecipitation and immunoblotting were carried out as described [22]. 


\section{$L C-M S / M S$}

The purified proteins bound on beads were directly digested with trypsin and analyzed by LC-MS/MS as we previously described [22].

\section{Results and discussion}

In order to identify both the enzymatic pathways and substrates for SUMO-2, SUMO-3, and NEDD8, we constructed mammalian expression vectors expressing the Myc-Histagged processed forms (UBL-GG) and the Myc-His-tagged non-conjugatable forms (UBL $\Delta \mathrm{GG}$ ) of these UBLs (Fig. 1). The dual tags strategy is used because relative to anti- $6 \times$ Histag antibody, the anti-Myc antibody is more sensitive for detecting Myc-containing proteins, and more efficient for immunoprecipitating the tagged proteins and their interacting proteins (data not shown). Therefore, purification of Myctagged UBLs under native condition could lead to the identification of the UBL pathway enzymes, target proteins and other interacting proteins. The purification of $6 \times$ His-tag UBLs under denaturing condition, on the other hand, minimizes the contamination by non-covalently interacting proteins, thus adding a level of confidence that the identified proteins are likely to be the bonafide target proteins.

The constructed vectors harboring these UBLs were transfected into HEK 293 Tet-On cells and stable cell lines were established using hygromycin selection, as described previously [22]. Myc-His-tagged UBL-GGs and UBL $\Delta$ GGs were successfully expressed in the stable cell lines as demonstrated by the Western analysis using anti-Myc antibody (Fig. 2). The epitope-tagged UBL-GGs could conjugate target proteins, as evident by the presence of high molecular weight protein smears formed in cells expressing Myc-HisUBL-GGs. In contrast, there were no detectable high molecular weight UBL conjugates formed in stable cell lines expressing Myc-His-UBL $\Delta$ GGs. The results indicated that the epitope-tagged UBLs were functional in stable cell lines and the $\mathrm{N}$-terminal tag did not appear to alter the conjugation capacity of the UBLs. Immunofluorescence showed that Myc-His-SUMO-2GG and Myc-His-SUMO-3GG and their conjugated proteins were distributed mainly in the nucleus and highly co-localized with PML (Fig. 3A), consistent with previous findings using YFP-SUMO chimeras [26]. In contrast, Myc-His-NEDD8GG was localized in both nucleus and cytosol (Fig. 3B). Since all of the cells stained positive for epitope-tagged proteins, we concluded that we have successfully established stable cell lines expressing Myc-His-SUMO2GG, Myc-His-SUMO-3GG or Myc-His-NEDD8GG.

\begin{tabular}{l|l|l|l|l}
\hline UBL-GG & Myc & $6 x H i s$ & UBL & GG \\
\hline
\end{tabular}

\begin{tabular}{|c|c|c|c|}
\hline UBL $\Delta \mathrm{GG}$ & Myc & 6xHis & $\overline{\text { UBL }}$ \\
\hline
\end{tabular}

Fig. 1. Schematic structures of epitope-tagged processed form (UBL-GG) and non-conjugatable form (UBL $\Delta \mathrm{GG}$ ) of ubiquitin-like modifiers (UBLs). Both Myc- and $6 \times$ His-tag were fused to the N-terminus of UBLGG and UBL $\Delta \mathrm{GG}$.

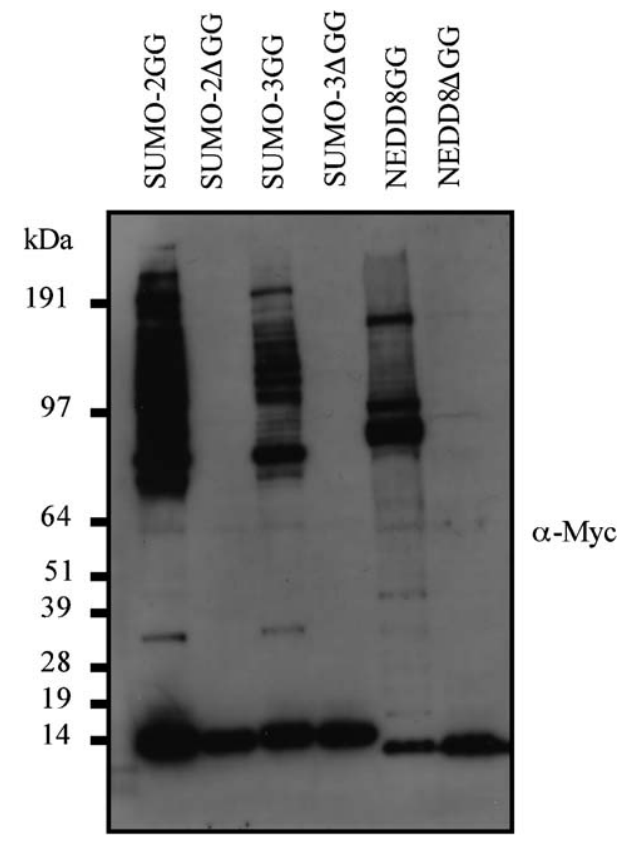

Fig. 2. Expression of epitope-tagged processed form (UBL-GG) and non-conjugatable form (UBL $\Delta$ GG) of UBLs in HEK 293 cells. HEK 293 Tet-On cells were transfected with pTRE2hyg2-Myc-His-SUMO-2/ 3GG, pTRE2hyg2-Myc-His-SUMO-2/3 $\Delta$ GG, pTRE2hyg2-Myc-His-N EDD8GG, or pTRE2hyg2-Myc-His-NEDD8 $\Delta$ GG expression plasmid, as indicated. Cells stably expressing UBL-GG or UBL $\Delta$ GG were selected with $300 \mu \mathrm{g} / \mathrm{ml}$ hygromycin. Following $48 \mathrm{~h}$ incubation with $2 \mu \mathrm{g} / \mathrm{ml}$ Dox, whole cell extracts were prepared and resolved in NuPAGE gels. Proteins were transferred to a PVDF membrane and probed with anti-Myc antibody.

A $\alpha-\mathrm{Myc}$ $\alpha-P M L$
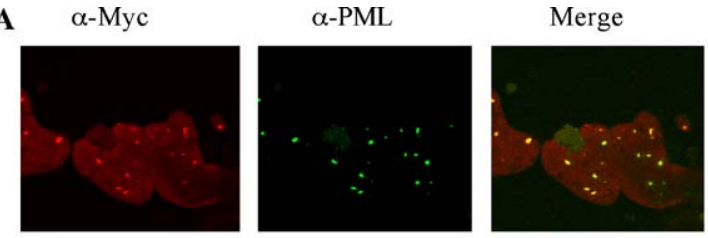

SUMO-2GG
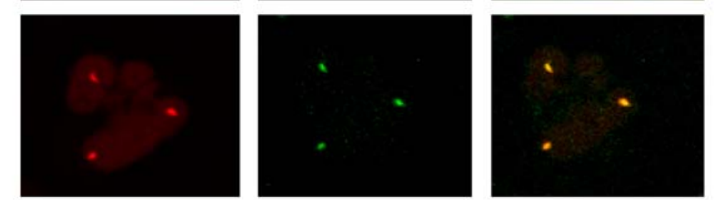

SUMO-3GG

B $\alpha$-Myc

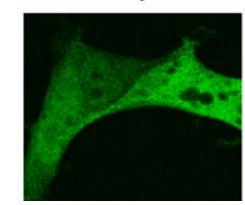

PI
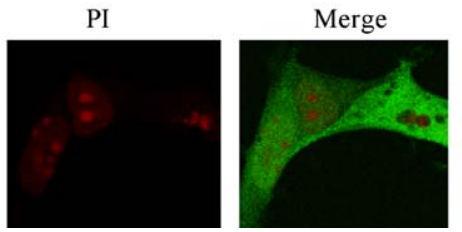

NEDD8GG

Fig. 3. Subcellular localization of SUMO-2GG, SUMO-3GG, and NEDD8GG. (A) Myc-tagged SUMO-2/3 and sumoylated proteins mainly distributed in the nucleus and highly co-localized with PML bodies. Following induction with $2 \mu \mathrm{g} / \mathrm{ml}$ Dox for $48 \mathrm{~h}$, HEK 293 Tet-On cells stably expressing SUMO-2/3GG were fixed with $3.7 \%$ formaldehyde and then stained with TRITC conjugated anti-Myc antibody and FITC conjugated anti-PML. (B) HEK 293 Tet-On cells stably expressing NEDD8GG were fixed and stained with anti-Myc primary antibody and then FITC conjugated secondary goat anti-mouse $\mathrm{IgG}$ antibody. The nuclei were stained with propidium iodide (PI). 
To analyze the enzymatic pathways for SUMO-2/3 and NEDD8, Myc-tagged SUMO-2/3GG, NEDD8GG, and their interacting proteins were purified under native conditions using anti-Myc conjugated agarose beads. The purified proteins were identified using LC-MS/MS methods. Unlike the singular subunit of E1 for ubiquitylation, there are two subunits for the E1-like activating enzymes for sumoylation and neddylation, one is homologous to the N-terminal domain of E1, and the other homologous to the C-terminal domain $[18,19]$. As shown in Table 1 , the two subunits of E1-like activating enzymes for SUMO-2 and NEDD8 pathways and one subunit of E1-like activating enzyme for SUMO-3 pathway were identified. In addition, all the E2like conjugating enzymes for SUMO-2/3 and NEDD8 pathways were also identified. These results indicate that this general method is useful for identifying the enzymatic pathways for UBLs. Interestingly, at least 20 peptides for SAE2 were identified for the SUMO-2 or SUMO-3 pathway, but only two peptides for SAE1 were identified for the SUMO-2 pathway. In addition, no peptide was found for the SAE1 of SUMO-3 pathway (Table 1). One plausible interpretation is that the putative active cysteine residue required to form a thioester bond with SUMO-2/3 is present in SAE2 [18]. This covalent linkage might allow SAE2 to be co-purified easily with SUMO-2/3. In contrast, SAE1 was only co-purified as a result of its interaction with SAE2.

To investigate the target proteins for these UBLs, we purified the $6 \times$ His-tagged SUMO-2/3GG, NEDD8GG, and their conjugated proteins under denaturing conditions using Ni-NTA agarose beads. The purified proteins were identified using LC-MS/MS. The $6 \times$ His-tagged proteins from cells expressing Myc-His-tagged SUMO-2 $\Delta \mathrm{GG}$, SUMO-3 $\Delta$ GG and NEDD8 $\Delta$ GG were also purified, and the identified proteins were used as negative controls. As shown in Table 1 , many previously confirmed target proteins for SUMO-2/3 pathways were identified, including
RanGAP1, RanBP2, and thymine DNA glycosylase [27-29]. Most of the known substrates for NEDD8 pathway were also identified, including 5 cullin family members, and p53 [30-32]. The neddylation of CUL1 and p53 proteins were further confirmed by Western analysis (Fig. 4B). The NEDD8 modified CUL1 or p53 was only detected in the Ni-NTA precipitate prepared from the NEDD8GG cell line, but not from the NEDD $8 \Delta \mathrm{GG}$ control cell line. The identification of these previously known substrates adds confidence to the efficiency of this method. Furthermore, the observed multi-neddylation of p53 (Fig. 4B, lower panel) is consistent with the findings that $\mathrm{p} 53$ contains three NEDD8 binding sites at its $\mathrm{C}$-terminus and p53 can be modified
A

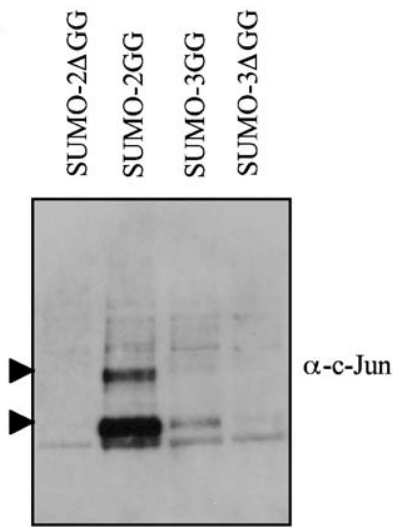

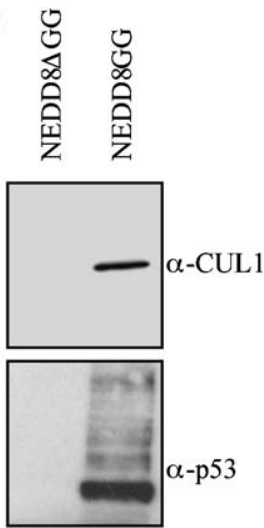

Fig. 4. (A) Western analysis of c-Jun in cell extracts. Whole-cell extracts from HEK 293 cells stably expressing SUMO-2/3DGG or SUMO-2/3GG were purified using Ni-NTA agarose beads. The purified proteins were resolved by a NuPAGE gel, transferred to a PVDF membrane and probed with anti-c-Jun antibody. The arrows indicate calculated molecular weights corresponding to those of mono- and bi-sumoylated c-Jun. (B) Similarly, the whole-cell extracts from HEK 293 cells stably expressing NEDD8DGG or NEDD8GG were purified using Ni-NTA agarose beads. The purified proteins were resolved by a NuPAGE gel, transferred to a PVDF membrane and probed with anti-CUL1 or anti-p53 antibody.

Table 1

SUMO-2/3 and NEDD8 pathway enzymes and substrates identified by LC-MS/MS

\begin{tabular}{|c|c|c|c|}
\hline & SUMO-2 & SUMO-3 & NEDD8 \\
\hline $\begin{array}{l}\text { Enzymes in pathway } \\
\text { (E1- and E2-like proteins) }\end{array}$ & $\begin{array}{l}\text { SAE1 }(\text { AAH1 } 8271,2) \\
\text { SAE2 }(\text { AAH03153, 21) } \\
\text { UBC9 }(\mathrm{P} 63279,2)\end{array}$ & $\begin{array}{l}\text { - } \\
\text { SAE2 }(A A H 03153,20) \\
\text { UBC9 }(\mathrm{P} 63279,4)\end{array}$ & $\begin{array}{l}\text { UBA3 }(\text { AAC27648, 7) } \\
\text { APPBP1 }(\text { Q13564, 7) } \\
\text { UBC12 }(\text { P61081, 5) }\end{array}$ \\
\hline Known substrates & $\begin{array}{l}\text { RanGAP1 }(\mathrm{P} 46060,18) \\
\text { RanBP2 (P49792, 4) } \\
\text { G/T mismatch-specific thymine } \\
\text { DNA glycosylase }(\mathrm{Q} 13569,1)\end{array}$ & $\begin{array}{l}\text { RanGAP1 }(\mathrm{P} 46060,17) \\
\text { RanBP2 }(\mathrm{P} 49792,5) \\
\text { G/T mismatch-specific thymine DNA } \\
\text { glycosylase }(\mathrm{Q} 13569,1)\end{array}$ & $\begin{array}{l}\text { CUL-1 (Q13616, 8) } \\
\text { CUL-2 (Q13617, 20) } \\
\text { CUL-3 (Q13618, 11) } \\
\text { CUL-4A (Q13619, 1) } \\
\text { CUL-4B (Q13620, 24) } \\
\text { P53 (P04637, 1) }\end{array}$ \\
\hline Unknown substrates & $\begin{array}{l}\text { CUL-3 }(\mathrm{Q} 13618,1) \\
\text { G1/S-specific cyclin E2 }(\mathrm{O} 96020,1) \\
\text { Bloom's syndrome protein }(\mathrm{P} 54132,1) \\
\text { Retinoblastoma-like protein 1 }(\mathrm{P} 28749,1) \\
\text { P53-binding protein 2 }(\mathrm{Q} 13625,1) \\
\text { Huntingtin-associated protein 1 }(\mathrm{O} 60229,1) \\
\text { c-Jun (CAG46552, 1) }\end{array}$ & $\begin{array}{l}\text { CUL-3 }(\mathrm{Q} 13618,1) \\
\text { G1/S-Specific cyclin E2 }(\mathrm{O} 96020,1) \\
\text { Bloom's syndrome protein }(\mathrm{P} 54132,1) \\
\text { Retinoblastoma-like protein } 1(\mathrm{P} 28749,1) \\
\text { P53-binding protein } 2(\mathrm{Q} 13625,1) \\
\text { Huntingtin-associated protein } 1(\mathrm{O} 60229,1) \\
\text { c-Jun (CAG46552, 1) }\end{array}$ & $\begin{array}{l}\text { PARP-1 (NP_001609, 2) } \\
\text { FAK (Q05397, 1) }\end{array}$ \\
\hline
\end{tabular}

Note. The protein accession number and the number of peptides identified by LC-MS/MS are shown in bracket and separated with a comma. 
simultaneously by NEDD8 and ubiquitin [32]. The elegant work of these authors also revealed that both ubiquitylation and neddylation of $\mathrm{p} 53$ is catalyzed by Mdm2 RING finger E3 ligase. Neddylation inhibits the transcriptional activity of p53 [32]. Since polyubiquitylation of protein leads to its degradation, neddylation of $\mathrm{p} 53$ provides an efficient and reversible regulatory mechanism for $\mathrm{p} 53$.

Meanwhile, seven previously unreported substrates were identified from both SUMO-2GG and SUMO-3GG cell lines (see Table 1). Among them, Bloom's syndrome protein and c-Jun have been previously shown to be the SUMO-1 substrates $[33,34]$. The modification of c-Jun by SUMO-2/3 was confirmed by Western analysis. Fig. 4A reveals that two protein bands, separated by $\sim 20 \mathrm{kDa}$, corresponding to the mono- and bi-sumoylated c-Jun were detected in the Ni-NTA precipitates from SUMO-2/3GG cell lines, but not from SUMO-2/3 $\Delta$ GG control cell lines. It has been reported that there are two SUMO-1 modification sites in c-Jun [35]. Therefore, it is likely that SUMO-2/3 may also modify c-Jun at these two sites. The difference in band densities probably reflects the difference in the levels of expression of SUMO-2GG, and SUMO-3GG as shown in Fig. 2. The rest of those putative SUMO-2/3 target proteins could not be confirmed by Western analysis. This is likely due to the low abundance of the proteins per se, or low levels of SUMO-2/3 modifications of these proteins, or the low affinity of antibodies available. Two newly identified NEDD8 modified proteins, PARP1, and FAK, were found (Table 1). However, they have yet to be confirmed by Western analysis under denaturing condition. It should be pointed out that although majority of the unknown substrates were identified by one peptide LC-MS/MS identification, the likelihood that these newly found substrates are UBL-modified proteins is supported by the observation that known substrates such as thymine DNA glycosylase, $\mathrm{Cul}-4 \mathrm{~A}$, and $\mathrm{p} 53$ were also confirmed via one-peptide identification. By overexpressing these putative target proteins together with the UBLs, it might be possible in the future to validate whether they are authentic substrates of the UBLs.

In summary, we have established a general and efficient method for identifying both the enzymatic cascades and major substrates for UBLs. The method relies on the enrichment of UBL-conjugated proteins, taking advantages of the dual tags introduced into recombinant UBLs, stable cell lines overexpressing the UBLs proteins, and LC-MS/ MS. Enrichment of UBL-protein provides a mean to overcome the problem of detecting low levels of modified proteins. Furthermore, immunoprecipitation using anti-Myc enables the identification of E1 and E2 for SUMO-2/3 and NEDD8 pathways and interacting proteins under native condition. Purification of $6 \times$ His-tagged proteins under denaturing conditions minimizes the contaminating proteins not covalently bound by UBLs. The analysis of proteins using LC-MS/MS provides an efficient and sensitive method, relative to Western blot analysis, for protein identification. This method is being applied to studying other UBLs, such as FAT10, and URM1.

\section{Acknowledgment}

This research was supported by the Intramural Research Program of the NIH, NHLBI.

\section{References}

[1] E.R. Stadtman, P.B. Chock, Curr. Top. Cell. Regul. 13 (1978) 53-95.

[2] A. Ciechanover, Cell 79 (1994) 13-21.

[3] A. Hershko, Trends Biochem. Sci. 21 (1996) 445-449.

[4] A.L. Haas, T.J. Siepmann, FASEB J. 11 (1997) 1257-1268.

[5] K.D. Wilkinson, FASEB J. 11 (1997) 1245-1256.

[6] M. Hochstrasser, Annu. Rev. Genet. 30 (1996) 405-439.

[7] C. Hoege, B. Pfander, G.L. Moldevan, G. Pyrowolakis, S. Jentsch, Nature 419 (2002) 135-141.

[8] Z.W. Sun, C.D. Allis, Nature 418 (2002) 104-108.

[9] M.D. Marmor, Y. Yarden, Oncogene 23 (2004) 2057-2070.

[10] S. Jentsch, G. Pyrowolakis, Trends. Cell. Biol. 10 (2000) 335-342.

[11] R.T. Hay, Mol. Cell 18 (2005) 1-12.

[12] K.J. Ritchie, D.E. Zhang, Semin. Cell Dev. Biol. 15 (2004) 237-246.

[13] D.T. Huang, H. Walden, D. Duda, B.A. Schulman, Oncogene 23 (2004) 1958-1971.

[14] R.L. Welchman, C. Gordon, R.J. Mayer, Nat. Rev. Mol. Cell Biol. 6 (2005) 599-609.

[15] E.S. Johnson, I. Schwienhorst, R.J. Dohmen, G. Blobel, EMBO J. 16 (1997) 5509-5519.

[16] L. Gong, E.T. Yeh, J. Biol. Chem. 274 (1999) 12036-12042.

[17] C. Zhao, S.L. Beaudenon, M.L. Kelley, M.B. Waddell, W. Yuan, B.A. Schulman, J.M. Huibregtse, R.M. Krug, Proc. Natl. Acad. Sci. USA 101 (2004) 7578-7582.

[18] J.M. Desterro, M.S. Rodriguez, G.D. Kemp, R.T. Hay, J. Biol. Chem. 274 (1999) 10618-10624.

[19] D. Liakopoulos, G. Doenges, K. Matuschewski, S. Jentsch, EMBO J. 17 (1998) 2208-2214.

[20] S. Raasi, G. Schmidtke, M. Groettrup, J. Biol. Chem. 276 (2001) 35334-35343.

[21] A.S. Goehring, D.M. Rivers, G.F. Sprague Jr., Mol. Biol. Cell 14 (2003) 4329-4341.

[22] T. Li, E. Evdokimov, R.F. Shen, C.C. Chao, E. Tekle, T. Wang, E.R. Stadtman, D.C. Yang, P.B. Chock, Proc. Natl. Acad. Sci. USA 101 (2004) 8551-8556.

[23] J. Peng, D. Schwartz, J.E. Elias, C.C. Thoreen, D. Cheng, G. Marsischky, J. Roelofs, D. Finley, S.P. Gygi, Nat. Biotechnol. 21 (2003) 921-926.

[24] V.G. Panse, U. Hardeland, T. Werner, B. Kuster, E. Hurt, J. Biol. Chem. 279 (2004) 41346-41351.

[25] N.V. Giannakopoulos, J.K. Luo, V. Papov, W. Zou, D.J. Lenschow, B.S. Jacobs, E.C. Borden, J. Li, H.W. Virgin, D.E. Zhang, Biochem. Biophys. Res. Commun. 336 (2005) 496-506.

[26] F. Ayaydin, M. Dasso, Mol. Biol. Cell. 15 (2004) 5208-5218.

[27] H. Saitoh, J. Hinchey, J. Biol. Chem. 275 (2000) 6252-6258.

[28] H. Zhang, H. Saitoh, M.J. Matunis, Mol. Cell Biol. 22 (2002) 6498-6508.

[29] U. Hardeland, R. Steinacher, J. Jiricny, P. Schar, EMBO J. 21 (2002) 1456-1464.

[30] F. Osaka, H. Kawasaki, N. Aida, M. Saeki, T. Chiba, S. Kawashima, K. Tanaka, S. Kato, Genes Dev. 12 (1998) 2263-2268.

[31] H. Wada, E.T. Yeh, T. Kamitani, Biochem. Biophys. Res. Commun. 257 (1999) 100-105.

[32] D.P. Xirodimas, M.K. Saville, J.C. Bourdon, R.T. Hay, D.P. Lane, Cell 118 (2004) 83-97.

[33] S. Eladad, T.Z. Ye, P. Hu, M. Leversha, S. Beresten, M.J. Matunis, N.A. Ellis, Hum. Mol. Genet. 14 (2005) 1351-1365.

[34] S. Muller, M. Berger, F. Lehembre, J.S. Seeler, Y. Haupt, A. Dejean, J. Biol. Chem. 275 (2000) 13321-13329.

[35] G. Bossis, C.E. Malnou, R. Farras, E. Andermarcher, R. Hipskind, M. Rodriguez, D. Schmidt, S. Muller, I. Jariel-Encontre, M. Piechaczyk, Mol. Cell Biol. 25 (2005) 6964-6979. 